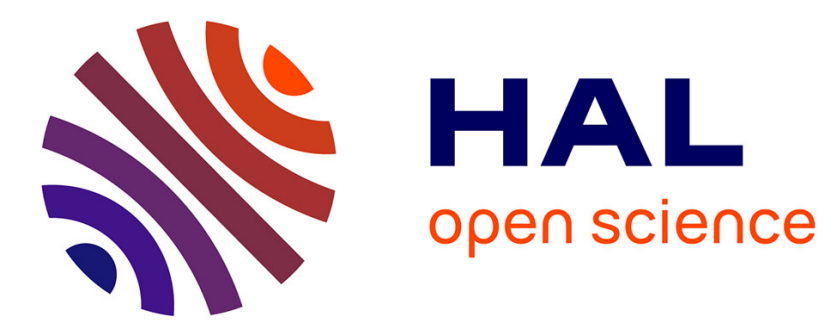

\title{
Vibration-based damage localization with load vectors under temperature changes
}

Md Delwar Hossain Bhuyan, Guillaume Gautier, Nicolas Le Touz, Michael Döhler, Falk Hille, Jean Dumoulin, Laurent Mevel

\section{- To cite this version:}

Md Delwar Hossain Bhuyan, Guillaume Gautier, Nicolas Le Touz, Michael Döhler, Falk Hille, et al.. Vibration-based damage localization with load vectors under temperature changes. Structural Control and Health Monitoring, 2019, 26 (11), pp.e2439. 10.1002/stc.2439 . hal-02293057

\section{HAL Id: hal-02293057 \\ https://hal.inria.fr/hal-02293057}

Submitted on 20 Sep 2019

HAL is a multi-disciplinary open access archive for the deposit and dissemination of scientific research documents, whether they are published or not. The documents may come from teaching and research institutions in France or abroad, or from public or private research centers.
L'archive ouverte pluridisciplinaire HAL, est destinée au dépôt et à la diffusion de documents scientifiques de niveau recherche, publiés ou non, émanant des établissements d'enseignement et de recherche français ou étrangers, des laboratoires publics ou privés. 
DOI: $\mathrm{xxx} / \mathrm{xxxx}$

\title{
RESEARCH ARTICLE
}

\section{Vibration-based damage localization with load vectors under temperature changes}

\author{
Md Delwar Hossain Bhuyan ${ }^{1}$ | Guillaume Gautier,2,3,4 | Nicolas Le Touz ${ }^{4,3}$ | Michael Döhler $2,3,4$ | Falk \\ Hille $^{1}$ | Jean Dumoulin ${ }^{4,3}$ | Laurent Mevel ${ }^{2,3,4}$
}

${ }^{1}$ BAM Federal Institute for Materials Research and Testing, Berlin, Germany

${ }^{2}$ Univ Rennes, Rennes, France

${ }^{3}$ Inria, Rennes, France

${ }^{4}$ Ifsttar, COSYS/SII, Bouguenais, France

\section{Correspondence}

Michael Döhler, Inria, Rennes, France.

Email: michael.doehler@inria.fr

\begin{abstract}
Summary
Damage detection and localization in civil or mechanical structures is a subject of active development and research. A few vibration-based methods have been developed so far, requiring for example modal parameter estimates in the reference and damaged states of the investigated structure, and for localization in addition a finite element model. For structures in operation, temperature has been shown to be a major nuisance to the efficiency of such methods since the modal parameters are varying not only with damage but also due to temperature variations. For detection, a few rejection approaches have been developed. Besides the increased complexity, environmental variation is hardly taken into account in localization approaches. In this paper, we propose a sensitivity-based correction of the identified modal parameters in the damaged state with respect to the temperature field in the reference state, based on a sensitivity analysis with respect to temperature dependent parameters of the finite element model in the reference state. The approach is then applied to the Stochastic Dynamic Damage Locating Vector (SDDLV) method, where its improved performance under non-uniform temperature variations is shown in a numerical application on a beam.
\end{abstract}

\section{KEYWORDS:}

damage localization, load vector, temperature effect rejection, temperature modeling, statistical evaluation

\section{1 | INTRODUCTION}

For civil engineering structures such as bridges, buildings or offshore structures, vibration-based Structural Health Monitoring (SHM) techniques have been actively developed for the last three decades ${ }^{1-4}$. The general aim of SHM in this context is the long-term measurement-based observation of the structural condition in the sense of damage development over time. The different tasks for damage diagnosis can be classified into five levels with increasing complexity 516 , namely damage detection, localization, classification, quantification and lifetime prognosis. For damage detection (level 1) a broad range of methods has been developed, utilizing diverse damage indexing features (e.g. $.177-10)$. While damage detection methods have reached some maturity for industrial applications, damage localization (level 2) methods are less developed so far 11 .

Damage localization methods can be classified into model-based methods, where a finite element $(\mathrm{FE})$ model of the structure is analyzed in connection with measurements, and into data-driven methods, where only the information from the measurements is used. Model-based methods update the parameters of an FE model of the healthy structure based on the measurements 
from the damaged system. Damage is located in those elements where parameters are modified ${ }^{12 \mid 13}$. Often however, modelbased methods are too poorly conditioned to be successful in practice, since the size of the finite element model is usually much larger than the dimension of the space containing the parameter that can be estimated from sparsely instrumented insitu SHM systems ${ }^{14}$. In data-driven methods ${ }^{2 / 4}$, the damage is located using only the recorded response of the structure. For example, damage localization can be performed based on changes in natural frequency ${ }^{15}$, mode shapes or modal curvature ${ }^{16}$, or modal strain energy ${ }^{17}$. Furthermore, artificial neural networks ${ }^{18119}$, genetic algorithms ${ }^{20}$, wavelet based analysis ${ }^{21}$ or other signal processing methods ${ }^{2223}$ are applied amongst others. Besides model-based and data-driven approaches, a third group of methods has emerged that combines properties of both approaches, using data-driven features computed in the reference and damaged states as well as information from a FE model of the healthy structure $\frac{11}{1}$. Then, damage indicators are defined with respect to the elements of the FE model, without updating it. For example, damage is located by a statistical analysis of a data-driven subspace-based damage residual with respect to model-based sensitivities ${ }^{2425}$, or by analyzing stress fields that are computed from data-driven load vectors and the FE model $26 \mid 28$.

This paper addresses the problem of vibration-based damage localization under temperature changes. It is well-known that not only physical changes due to structural damage induce changes in the dynamic response, but the structural dynamics are also affected by environmental and operational influences, e.g. temperature and humidity or traffic and wind loading 29,31 . Especially varying temperature may affect damage features far more significantly than local structural damage in early stage $32 \mid 34$. The temperature distribution may be non-uniform over the structure ${ }^{35536}$. To eliminate those damage masking effects, different concepts have been proposed in the last decades. Generally, two different approaches are pursued to eliminate the temperature effect on the structural response. Physical model-based procedures are closely connected with the structural model and employ optimization algorithms to solve the inverse problem including the temperature effect ${ }^{37}$. On the other hand, statistical methods are often based on measurement data only without using any geometrical or material information, often without even using temperature measurements, but a database under varying conditions instead. To eliminate the effect of perturbations in damage indicating features, the data is processed utilizing statistical models that describe empirically the temperature influence e.g. on modal parameters and eliminate their effect using e.g. regression analysis with ARX models ${ }^{29134}$, Gaussian mixture models ${ }^{38}$, factor analysis ${ }^{2930}$, principal component analysis ${ }^{7 / 16}$, cointegration ${ }^{39}$ and others. Other statistical methods take into account physical properties of the temperature effect on the structure based on physical models ${ }^{40}$. However, such methods have mainly been applied in the context of damage detection, but hardly on damage localization so far.

In this paper, we develop an approach for the rejection of temperature variations on the modal parameters in connection with a damage localization approach, namely the Stochastic Dynamic Damage Locating Vector (SDDLV) ${ }^{26}$, which operates on the identified modal parameters in the reference and damaged states of the structure. We propose to reject possible temperature variations in a preprocessing step by a sensitivity-based correction of the modal parameter estimates in the damaged state with respect to the temperature in the reference state. The method allows to take into account non-uniform temperature fields on the structure by using temperature measurements at several locations, e.g. by means of an infrared camera, which avoids in particular the simplifying assumption of just one measured global temperature. We will show that the performance of the damage localization method is significantly improved by the proposed temperature correction strategy when applying it to simulated vibration data on a beam structure under non-uniform temperature variations.

The paper is organized as follows. In Section 2 an outline of the proposed damage localization method under temperature variations is presented. In Section 3 the SDDLV method is recalled and the rejection of the temperature effect is developed. The temperature model for data simulation is presented in Section 4 . Finally, in Section 5 the proposed method is applied on a numerical aluminum beam and its performance is evaluated.

\section{2 | OUTLINE OF THE TEMPERATURE CORRECTION METHOD}

The proposed temperature correction method is suited for combined data-driven and model-based localization approaches, for which modal parameter estimates are available in the reference and damaged states of the structure, as well as an FE model of the structure in the reference state. In particular, the SDDLV method ${ }^{26}$ is such an approach. To localize damage with this approach, a certain external load is computed from the modal parameters in both states that has the property that the resulting stress field at the damaged elements is zero when applying this load to the FE model. In subsequent work ${ }^{41,43}$, the statistical SDDLV (SSDDLV) approach has been proposed, taking into account uncertainties in the stress computation due to noisy measurement data. Though, considering the FE model and measurements in the reference state to be taken at some reference temperature 


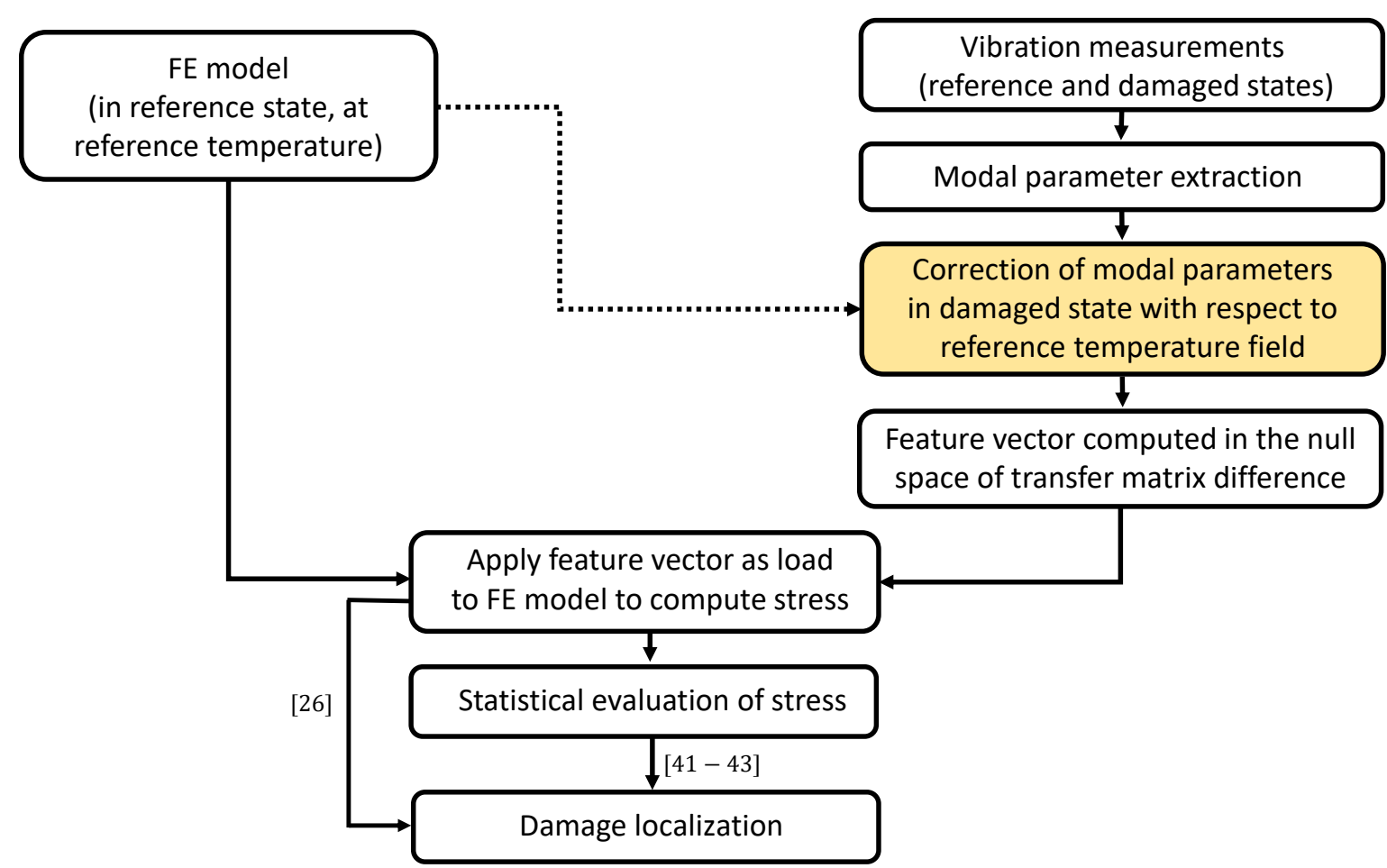

FIGURE 1 Proposed SDDLV-based damage localization strategy with temperature correction.

conditions, the modal parameters in the damaged state may be affected by variations from the reference temperature. So far, such environmental variability is not taken into account in the approach.

To correct the modal parameter estimates in the damaged state, first a temperature sensitivity analysis is carried out in the reference state, where the derivative of the modal parameters with respect to the temperature in the different elements of the FE model is obtained based on the material properties of the elements. Then, using actual temperature measurements in the damaged state, the modal parameters will be corrected with respect to the reference temperature field based on the sensitivity from the reference state. A scheme of the proposed approach is depicted in Figure 1

For a numerical application, a non-uniform temperature field will be simulated using a heat flux model for temperature propagation. It is then applied to a numerical beam for the simulation of vibration data. Note that the proposed temperature correction scheme only requires the measurement of temperatures over some of the elements of the FE model. In this way, information on the temperature model is not needed for the method itself.

\section{3 | TEMPERATURE EFFECT REJECTION FOR DAMAGE LOCALIZATION WITH S-SDDLV}

In this section, the underlying dynamical models are recalled and the temperature correction approach is developed for the estimates of the modal parameters in the damaged state. Then, the approach is applied to the SDDLV method and its statistical counterpart, the S-SDDLV, for temperature robust damage localization.

\section{1 | Dynamic models of a structure}

The behavior of a mechanical structure can be described by a linear time-invariant (LTI) dynamic system

$$
\mathcal{M} \ddot{\mathcal{X}}(t)+\mathcal{C} \dot{\mathcal{X}}(t)+\mathcal{K} \mathcal{X}(t)=f(t)
$$


where $\mathcal{M}, \mathcal{C}, \mathcal{K} \in \mathbb{R}^{d \times d}$ are the mass, damping and stiffness matrices, respectively, $t$ indicates continuous time and $\mathcal{X} \in \mathbb{R}^{d}$ denotes the displacements at the $d$ degrees of freedom (DOF) of the structure. The external force $f(t)$ is not measurable and modeled as white noise. Let the dynamic system (1) be observed at $r$ coordinates. Defining $x=\left[\begin{array}{ll}\mathcal{X} & \dot{\mathcal{X}}\end{array}\right]^{T}$, this leads to the corresponding continuous-time state-space model

$$
\left\{\begin{array}{c}
\dot{x}(t)=A x(t)+v(t) \\
y(t)=C x(t)+w(t)
\end{array}\right.
$$

with state vector $x \in \mathbb{R}^{n}$, output vector $y \in \mathbb{R}^{r}$, process noise $w \in \mathbb{R}^{n}$, output noise $v \in \mathbb{R}^{r}$, the state matrix $A \in \mathbb{R}^{n \times n}$ and output matrix $C \in \mathbb{R}^{r \times n}$, where $n=2 d$ is the system order. The system matrices of the discrete-time system corresponding to

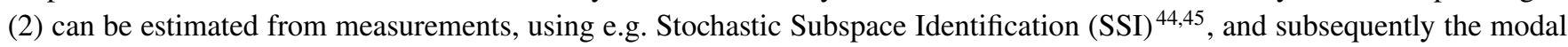
parameter estimates. Since not only structural modes, but also spurious modes appear when identifying the modal parameters, the latter should be discarded in a stabilization diagram, and finally estimates of $A$ and $C$ are obtained from the selected modal parameters. Details for their computation for the SDDLV approach are given in ${ }^{42}$.

\section{2 | Temperature correction of modal parameters}

Since the considered damage localization approach operates on the modal parameter estimates, it is convenient to reject the temperature effect directly on the modal parameters. We propose a sensitivity-based rejection approach, using temperature measurements on the structure in the reference and damaged states. In this way, the modal parameters in the damaged state will be approximated under the same temperature conditions as in the reference state. Hereby several temperature measurements at different positions on the structure can be used, e.g. from several temperature sensors or infrared cameras, assuming a possibly non-uniform temperature field.

Assume that $T \in \mathbb{R}^{p}$ is a temperature parameter vector that describes the monitored system in the current state, and $T_{0} \in \mathbb{R}^{p}$ its value in the reference system for $p$ temperature measurement points. In the following, the sensitivity matrix is obtained of the modal parameters with respect to $T$, i.e. the derivative of the eigenvalues $\lambda_{j}$ and mode shapes $\varphi_{j}$ related to $A$ and $C$ for $j=1, \ldots, m$. Denote $\Lambda=\left[\begin{array}{lll}\lambda_{1} & \ldots & \lambda_{m}\end{array}\right]^{T}$ and $\Phi=\left[\begin{array}{lll}\varphi_{1} & \ldots & \varphi_{m}\end{array}\right]$ the collection of eigenvalues and mode shapes, respectively, and let

$$
\theta=\left[\begin{array}{c}
\Lambda \\
\operatorname{vec}(\Phi)
\end{array}\right]
$$

be the full modal parameter vector of the system. These parameters are temperature dependent, $\theta=\theta(T)$. The first-order Taylor expansion of the parameter vector writes as

$$
\theta(T) \approx \theta\left(T_{0}\right)+\mathcal{J}_{\theta, T}\left(T-T_{0}\right),
$$

where $\mathcal{J}_{\theta, T}=\left.\frac{\partial \operatorname{vec}(\theta)}{\partial \operatorname{vec}(T)}\right|_{T=T_{0}} \in \mathbb{C}^{m(r+1) \times p}$ is the sensitivity matrix. Then, the rejection of temperature variation from the considered parameter vector $\theta(T)$ in an arbitrary state can be obtained from (3) as

$$
\theta\left(T_{0}\right) \approx \theta(T)-\mathcal{J}_{\theta, T}\left(T-T_{0}\right) .
$$

In the following section, the sensitivity $\mathcal{J}_{\theta, T}$ of the modal parameters with respect to temperature is derived.

\subsection{1 | Sensitivities of the modal parameters}

Assuming that only the stiffness of the system is affected by temperature variations, i.e. $\mathcal{K}=\mathcal{K}(T)$, the derivative $\mathcal{J}_{\theta, T}$ of the modal parameters with respect to the temperature vector can be obtained through the derivative of the stiffness matrix with respect to the temperature vector. The eigenvalue and mode shape derivatives with respect to any component $T_{k}$ of the temperature vector $T$ yield 46

$$
\begin{aligned}
& \frac{\partial \lambda_{j}}{\partial T_{k}}=-\frac{1}{a_{j}} \phi_{j}^{T} \frac{\partial \mathcal{K}(T)}{\partial T_{k}} \phi_{j}, \\
& \frac{\partial \varphi_{j}}{\partial T_{k}}=\sum_{i=1, i \neq j}^{m} \frac{1}{a_{i}} \frac{1}{\lambda_{i}-\lambda_{j}} \phi_{i}^{T} \frac{\partial \mathcal{K}(T)}{\partial T_{k}} \phi_{j} \varphi_{i}+\sum_{i=1}^{m} \frac{1}{a_{i}^{*}} \frac{1}{\lambda_{i}^{*}-\lambda_{j}} \phi_{i}^{H} \frac{\partial \mathcal{K}(T)}{\partial T_{k}} \phi_{j} \varphi_{i}^{*}
\end{aligned}
$$

where $a_{i}=2 \lambda_{i} \phi_{i}^{T} \mathcal{M} \phi_{i}+\phi_{i}^{T} \mathcal{C} \phi_{i}$, "**" denotes the complex conjugate and " $H$ " the conjugate transpose, and $\phi_{i}$ are the eigenvectors of system (1) at all DOFs. Note that $\varphi_{i}$ is equal to the components of $\phi_{i}$ at the sensor DOFs. The relation between the temperature 
component $T_{k}$ and the material properties of the structure is used to obtain the derivative $\frac{\partial \mathcal{K}(T)}{\partial T_{k}}$, e.g. using a finite difference approach. Assembling the real and imaginary parts of (5) and (6) for $j=1, \ldots, m$ in the rows and for $k=1, \ldots, p$ in the columns leads to the sensitivity matrix $\mathcal{J}_{\theta, T}$.

\subsection{Computation of the temperature-robust damage indicator}

The S-SDDLV damage indicator $26141-43]$ is based on the transfer matrix difference $\delta G(s)$ between reference and damaged states. The transfer function $G(s) \in \mathbb{C}^{r \times r}$ of the system at the sensor coordinates can be derived from (2) as 26

$$
G(s)=R(s) D \text { where } R(s)=C(s I-A)^{-1}\left[\begin{array}{c}
C A \\
C
\end{array}\right]^{\dagger}\left[\begin{array}{l}
I \\
0
\end{array}\right]
$$

under the condition that the system order satisfies $n \leq 2 r$, where $D$ is the theoretical direct transmission matrix of the state space model that however is not required in the following. As outlined in the previous section, we assume the system in the reference state under (reference) temperature vector $T_{0}$, and in the damaged state under an arbitrary temperature $T$. The identified modal parameters in the damaged state are then corrected with the temperature correction approach in order to approximate their behavior at $T_{0}$. Subsequently, all computations in the damaged state are made with the corrected modal parameters.

The difference between the transfer matrices in both damaged (variables with tilde) and healthy states is $\delta G(s)=\tilde{G}(s)-G(s)$, where $\tilde{G}(s)$ in the damaged state is obtained with the temperature correction approach. Damage is considered as changes in the stiffness of system (1), while mass remains unchanged. In the SDDLV approach, a vector in the null space of $\delta G(s)$ is obtained, which is identical to the null space of $\delta R(s)^{T}=\tilde{R}(s)^{T}-R(s)^{T 26}$. Then, the desired load vector $v(s) \in \mathbb{C}^{r}$ can be obtained from the null space of $\delta R(s)^{T}$ using a singular value decomposition (SVD). Finally, the load $v(s)$ is applied to the FE model of the healthy structure at the temperature $T_{0}$ to compute the stress field over all structural elements. The respective stress resultants for each of the elements are stacked in vector $S(s)$, which yields a linear relationship to the load that can be expressed by a matrix $L_{\text {model }}(s) \in \mathbb{C}^{l \times r}$ based on the FE model of the structure, satisfying

$$
S(s)=L_{\text {model }}(s) v(s) .
$$

The stress vector $S(s)$ indicates potential damage for elements with corresponding entries in $S(s)$ that are close to zero. When estimated, these stresses are not exactly zero but small in practice because of modal truncation, model errors and statistical uncertainties due to measurement data. For increased robustness, the evaluation of the stress can be done at several values of $s$ and several mode sets 4243 .

To take into account estimation uncertainties that are due to the measurement data, a statistical evaluation of the stress is carried out. The covariance of the stress vector $\Sigma_{S}$ has been estimated in 41,43 . Then, an appropriate hypothesis test is performed on each structural element $t$ by selecting the respective stress components $S_{t}$ in $S(s)$ as well as the respective covariance submatrix $\Sigma_{t}$ of $\Sigma_{S}$, and computing the test statistic

$$
\chi_{t}^{2}=S_{t}^{T} \Sigma_{t}^{-1} S_{t}
$$

for each structural element $t$ tested for damage. Since stress over damaged elements is zero in theory, potential damage is located in the elements $t$ corresponding to the lowest value of $\chi_{t}^{2}$ among all elements.

Notice that the correction of the modal parameters with respect to the effect of temperature variation is a deterministic step. In this sense, the covariances of the modal parameters after correction are not affected and do not need to be corrected. Still, the values of the modal parameters go into the computation of the covariances of the transfer functions, the load vector and then the stress. Thus, these covariances are affected by the temperature correction strategy and the modal parameter values after correction are used in their computation.

\subsection{Summary of the algorithm}

The proposed algorithm of the damage localization approach runs as follows with the proposed temperature correction strategy.

1. The FE model of the structure is obtained at reference temperature vector $T_{0}$, and matrix $L_{\text {model }}(s)$ is extracted from it for the stress computation (Equation (7))

2. Vibration measurements are recorded in the reference state at reference temperature $T_{0}$. The modal parameters are identified from these measurements and used for estimation of the system matrices $\widehat{A}$ and $\hat{C}$ in the reference state, and their covariance is obtained (see $\left.{ }^{42}\right)$. 
3. Based on the FE model and a relation between the temperature vector $T$ and the material properties of the structure, the sensitivity $\mathcal{J}_{\theta, T}$ of the modal parameters with respect to temperature changes is obtained (see Equation (3)).

4. Vibration measurements are recorded in the damaged state at an arbitrary temperature vector $T$. The estimates of the modal parameters are obtained and corrected using the temperature correction strategy (4) and subsequently, the matrices $\widehat{A}$ and $\widehat{C}$ are computed in the damaged state rejecting the temperature variation by the proposed sensitivity analysis.

5. In order to compute the stress field $S(s)$ in 77 for damage localization, the load vector $v(s)$ in the null space of the transfer matrix difference $\delta G(s)$ between the healthy and damaged systems is applied to the FE model corresponding to the structure in the reference state at the reference temperature. This evaluation can be done at several values of $s$ and several mode sets $\left(\operatorname{see}^{\sqrt{42 / 43}}\right)$.

6. Finally, the statistical evaluation of the stress field is carried out in the $\chi^{2}$ tests in (8), and damage is localized at the lowest test values.

\section{4 | THE NUMERICAL TEMPERATURE MODEL}

The numerical temperature model aims at computing the temperature field distribution inside the structure from the knowledge of the environmental conditions. This is required for the simulation of relevant temperature scenarios in the numerical application that go beyond simple uniform temperature distributions. It is here supposed that the investigated structure is submitted to:

- convective heat exchange with the air,

- radiative heat exchange with the environment,

- outside energy contributions (such as solar radiation or other active thermal source).

The temperature evolution in the structure is governed by the heat diffusion equation, which can be written as $\frac{47}{47}$

$$
\rho c \frac{\partial T}{\partial t}-\nabla \cdot(k \nabla T)=q,
$$

where $\rho$ refers to the density, $c$ is the thermal capacity, $k$ is the thermal conductivity and $q$ is a thermal source. $\rho, c$ and $k$ can be temperature dependent. The boundary conditions are defined as follows:

$$
k \nabla T \cdot \vec{n}=h_{c}\left(T_{a}-T\right)+\varepsilon \sigma\left(T_{e}^{4}-T^{4}\right)+\Phi,
$$

where $\vec{n}$ is the outside normal, $h_{c}$ the convective exchange coefficient, $\varepsilon$ the emissivity, $\sigma=5.67 \times 10^{-8} \mathrm{~W} \cdot \mathrm{m}^{-2} \cdot \mathrm{K}^{-4}$ the Stefan constant, $T_{a}$ the air temperature, $T_{e}$ the environmental temperature and $\Phi$ the total outside contribution.

In outdoor conditions, the value of the convective heat exchange coefficient depends on the wind speed. As an example, MacAdams correlation estimates $h_{c}$ as $5.7+3.8 V_{w}$ for wind speed $V_{w} \leq 5.0 \mathrm{~m} . \mathrm{s}^{-1448}$. A temperature difference of about $10^{\circ} \mathrm{C}$ between the structure and the air with a null wind speed also entails a total heat flux of about $60 \mathrm{~W} \cdot \mathrm{m}^{-2}$.

For radiative exchange with the environment, $T_{e}$ is the sky temperature if the structure is located outdoors, with a large part of the sky visible, or the wall temperature for the case the structure is located indoors. As a first approximation, the sky temperature can be taken as $T_{e}=T_{a}-6^{49}$. For the case of a structure temperature at the surface $(\varepsilon \approx 1)$ equal to $20^{\circ} \mathrm{C}$ exposed to air at temperature $10^{\circ} \mathrm{C}$, the sky temperature can be estimated as $4^{\circ} \mathrm{C}$ and the heat flux coming from radiative transfer is also about $80 \mathrm{~W} . \mathrm{m}^{-2}$. Outdoors, during the daylight period, the main thermal effect comes also from solar radiation, with a heat flux that can reach $1000 \mathrm{~W} . \mathrm{m}^{-2}$ at solar noon, close to summer solstice. Even during the winter period, solar heat flux still reaches 300 W. $\mathrm{m}^{-2}$ in France.

It is moreover supposed that the initial temperature field in the structure is known (or at least estimated) in the numerical model, i.e.

$$
T(t=0)=T_{0}
$$

Applying the finite element method allows to get the following system of equations $\frac{50}{50}$ :

$$
C \dot{T}(t)+K(t) T(t)=F(t),
$$


where $C$ is the thermal capacity matrix, $K$ is the thermal conductivity matrix and $F$ the thermal source vector. $F$ depends on environmental conditions, $K$ is a function of the thermal properties of the structure and of the environmental conditions, while $C$ depends only on the thermal properties of the structure.

Equation (9) is solved in time with the Crank-Nicolson method, which allows to get an order 2 accuracy for time dependence. The temporal scheme is

where $l$ refers to the $l^{\text {th }}$ time step.

$$
T^{l+1}=\left(\frac{M}{\Delta t}+\frac{K}{2}\right)^{-1}\left[\left(\frac{M}{\Delta t}-\frac{K}{2}\right) T^{l}+\frac{1}{2}\left(F^{l}+F^{l+1}\right)\right],
$$

In this way, the temperature field over a structure can be obtained at a certain point in time. For the numerical application, this allows us 1) to simulate the temperature vector $T$ at the desired coordinates within the temperature field, and 2) to simulate vibration data according to the temperature field after obtaining the Young modulus of each element of the FE model based on the material properties of the element at its respective temperature.

\section{5 | NUMERICAL APPLICATION}

The proposed method has been applied on a 3D rectangular beam model in order to investigate the temperature effect and its rejection on the dynamic properties of the structure in the computation of the stress and its statistical evaluation for damage localization. The temperature model introduced in the previous section has been applied to the beam to simulate different temperature scenarios.

In this application, the outcome of the damage localization results with temperature correction is compared to not using any temperature correction, and the performance of the proposed method is evaluated using Monte Carlo simulations.

\section{1 | Beam model}

A 3D aluminum beam model, matching a previously laboratory-designed and experimentally-realized mock-up ${ }^{51}$, has been considered for damage localization under the temperature effect. The thickness and nature of the beam elements satisfy the hypothesis of a Biot number lower than 0.1, which satisfies the assumption of a homogeneous temperature distribution independent of the thickness at each time step. For instance, in such an experimental configuration, a thermal infrared camera may be used to identify the temperature distribution along the beam elements ${ }^{52}$. The structure is modeled with 4 main beam elements of length $1 \mathrm{~m}$. The elements are modeled as rectangular hollow structural sections (HSS) with internal $0.142 \mathrm{~m} \times 0.042 \mathrm{~m}$ and external $0.15 \mathrm{~m} \times 0.05 \mathrm{~m}$ sections, as depicted in Figure 2 The mass density, Young modulus and Poisson ratio are $2700 \mathrm{~kg} . \mathrm{m}^{-3}$, $70 \mathrm{GPa}\left(\right.$ at $\left.20^{\circ} \mathrm{C}\right)$ and 0.33 , respectively. Each section is enclosed by two plates of length $0.01 \mathrm{~m}$ with external $0.18 \mathrm{~m} \times 0.08 \mathrm{~m}$ and internal $0.142 \mathrm{~m} \times 0.042 \mathrm{~m}$ sections, respectively. Finally, at the beam ends, two plates of length $0.02 \mathrm{~m}$ and section $0.3 \mathrm{~m} \times$ $0.08 \mathrm{~m}$ are mounted. All beam sections have the same physical properties as the main beam elements. Each main beam element is discretized into 5 small elements and hence, the total number of elements is 30 , and the number of degrees of freedom of the structure is 180 . Damping is defined such that the damping ratio of all modes is $0.2 \%$.

The reference temperature of the beam is $20^{\circ} \mathrm{C}$. According to the considered temperature scenarios in the following, an additional heat flux is applied at element 17 of the beam with the temperature model introduced in Section 4 leading to a non-uniform temperature field of the beam. Then, the Young modulus of each beam element is set according to the obtained temperature in the element based on the material properties of aluminum ${ }^{53}$, and the resulting FE model is used for data simulation.

Two damage scenarios are considered. First, damage is modeled at elements $12-14$ (1.45 m from left end) by a $30 \%$ stiffness loss. Second, damage is located at elements 20-21 (2.87 m from left end) with a $25 \%$ and $45 \%$ stiffness loss, respectively. For the damaged and undamaged states, the acceleration data length for each simulated set is $N=100,000$ at a sampling frequency of $2000 \mathrm{~Hz}$, generated from collocated white noise excitation using four sensors in the Y-direction at $0.43 \mathrm{~m}, 1.49 \mathrm{~m}, 2.49 \mathrm{~m}$, and $3.47 \mathrm{~m}$ from the left support end. White measurement noise with $5 \%$ of the signal's standard deviation was added to the output data. An overview of the FE model of the beam with the sensor positions, heat flux location and damaged elements is given in Figure 3 


\section{Top view}

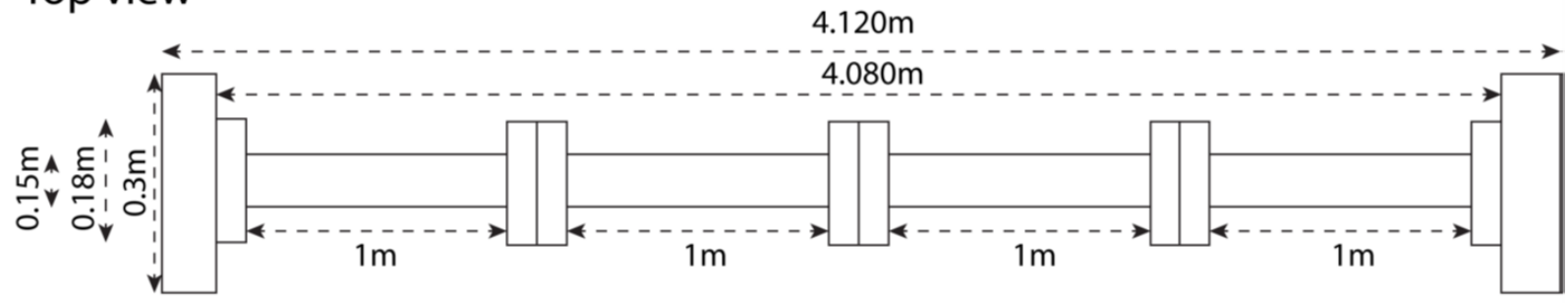

\section{Sections}
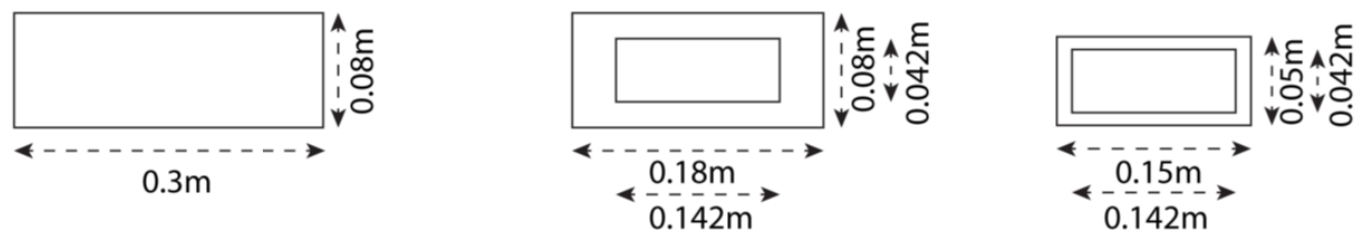

FIGURE 2 Dimensions of the beam model.

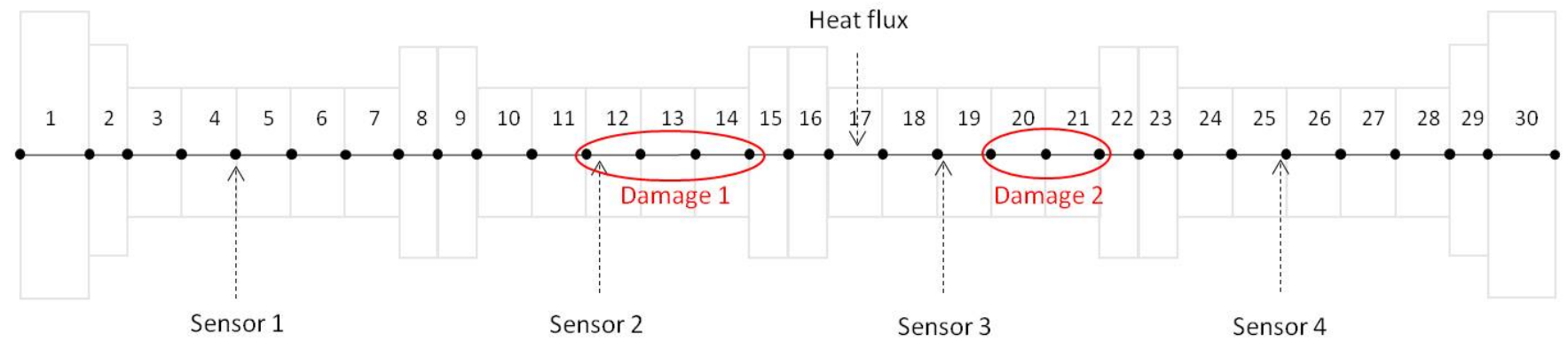

FIGURE 3 Elements of the beam model, including sensor positions, heat flux location and damaged elements.

\subsection{1 | Modal analysis}

In the SDDLV approach, the number of considered modes cannot be higher than the number of sensors. Using four sensors, the first four modes of the structure are identified from a simulated dataset using stochastic subspace identification (SSI), see Table 1. The identified mode shapes of the first four modes are illustrated in Figures 4 and 5 for the healthy and damaged states together with the mode shapes obtained from the FE model in the healthy state. Moreover, Figure 6(a)-(b) shows how the first and second modal frequencies are varying in terms of increasing heat flux for both healthy and damaged conditions.

TABLE 1 Frequency $(f[\mathrm{~Hz}])$ from healthy state (reference temperature $20^{\circ} \mathrm{C}$ ) and damaged states (heat flux $40 \mathrm{~W} \cdot \mathrm{m}^{-2}$ ).

\begin{tabular}{rrr}
\hline Mode & Healthy state & Damaged state \\
\hline 1 & 22.14 & 21.03 \\
2 & 61.10 & 59.00 \\
3 & 118.6 & 113.3 \\
4 & 204.5 & 194.2 \\
\hline
\end{tabular}




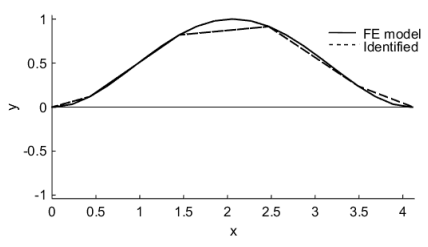

(a) First mode

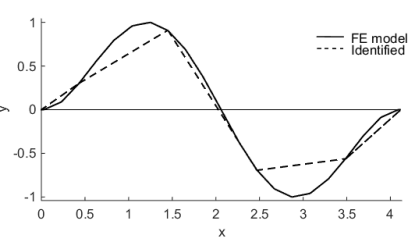

(b) Second mode

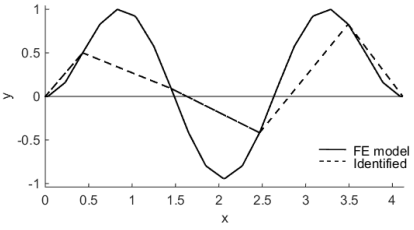

(c) Third mode

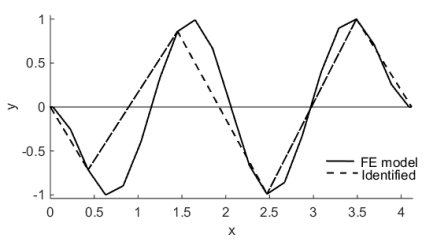

(d) Fourth mode

FIGURE 4 Mode shapes of the first four vertical modes in the healthy state from data and mode shapes from FE model in healthy state.

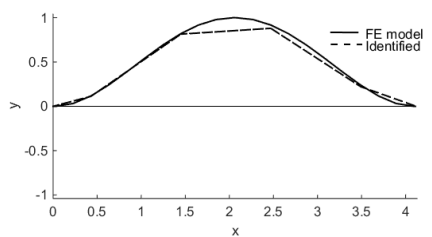

(a) First mode

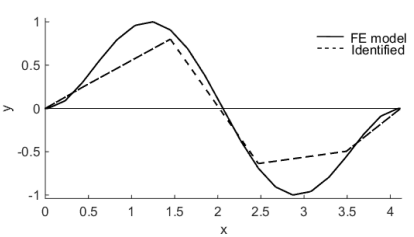

(b) Second vertical mode

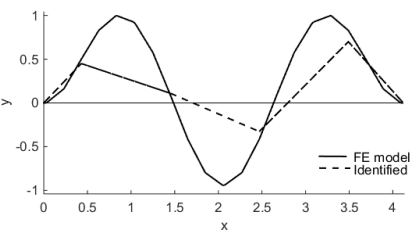

(c) Third mode

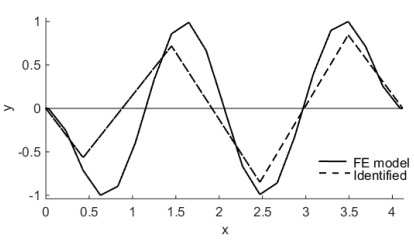

(d) Fourth mode

FIGURE 5 Mode shapes of the first four vertical modes in the damaged state (30\% stiffness loss) from data and mode shapes from FE model in healthy state.

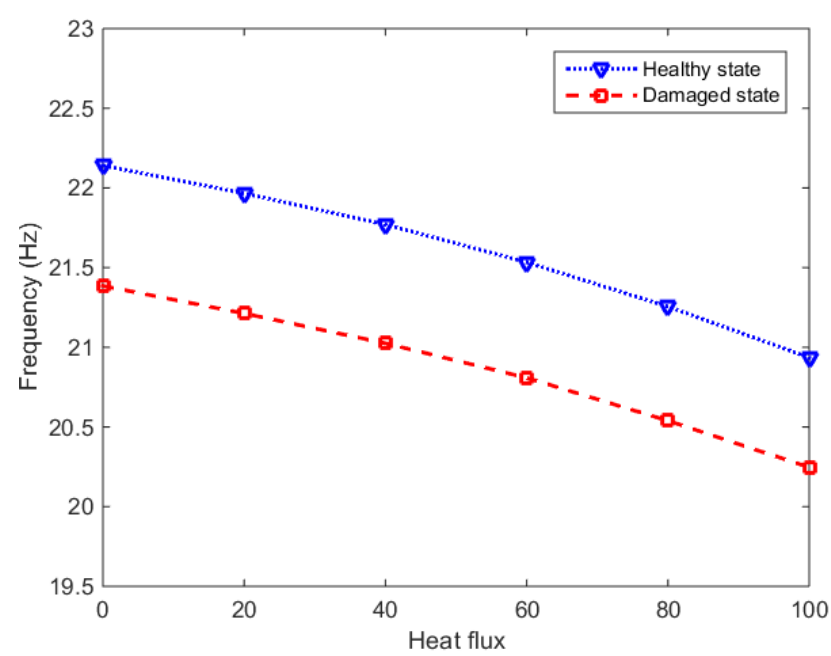

(a) First frequency

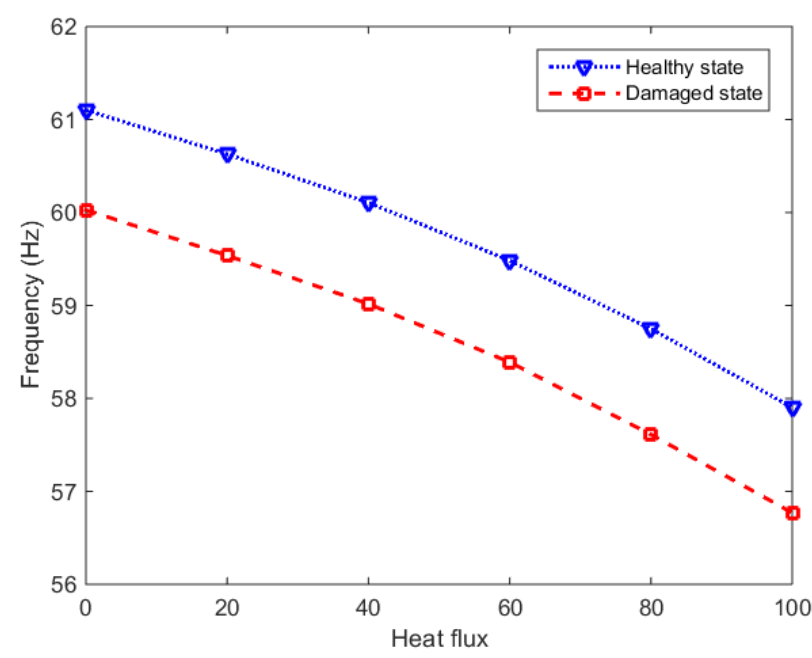

(b) Second frequency

FIGURE 6 Temperature effect on the first (a) and second (b) frequency for both healthy and damaged states. Damaged elements are 12,13 and 14 with $30 \%$ stiffness loss.

\subsection{Localization results in all elements using one dataset}

To analyze the impact of temperature variation on both the stress computation and its statistical evaluation for damage localization, results for each element are illustrated in Figures 7 and 8 taking into account temperature correction or not. The values for the Laplace variable $s$ is chosen as $s_{1}=-1+700 \mathrm{i}$ in the vicinity of the identified poles. In the following, first, results are illustrated considering no temperature correction and then results are illustrated using the temperature correction strategy. To compare the ratios between test results for the healthy and damaged elements, the computed test values are normalized in the figures such that the smallest of the 30 values - indicating damage - is 1 . 


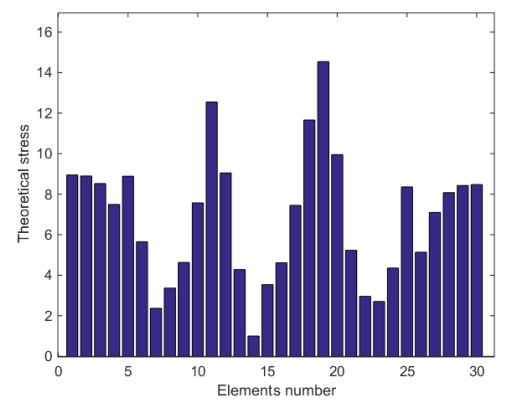

(a) Theoretical stress

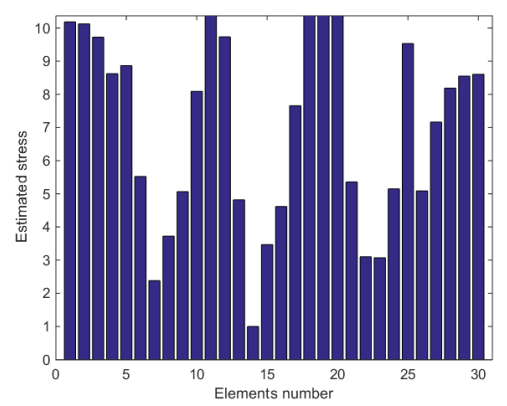

(b) Estimated stress

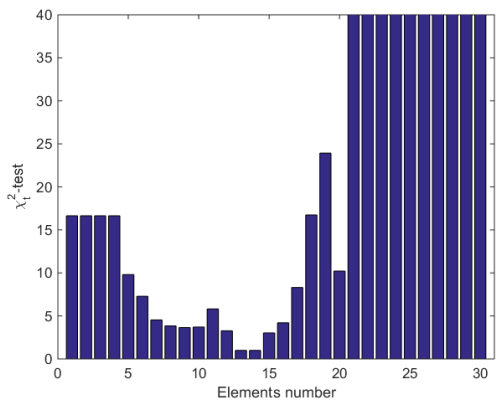

(c) Statistical test

FIGURE 7 Without temperature correction - localization results at all elements for stress computation and its statistical evaluation using one dataset and one $s$-value $(s=-1+700 \mathrm{i})$. Damaged elements are 12, 13 and 14 with stiffness loss of $30 \%$.

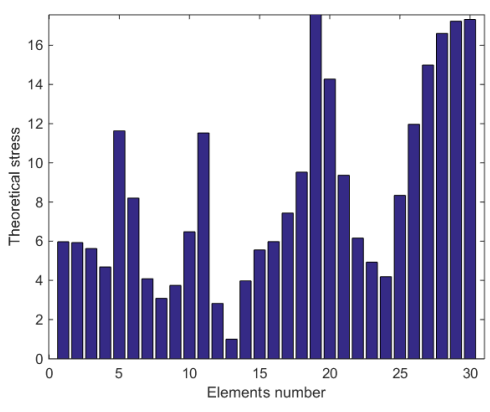

(a) Theoretical stress

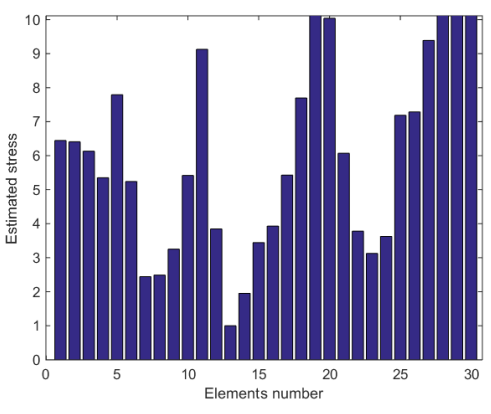

(b) Estimated stress

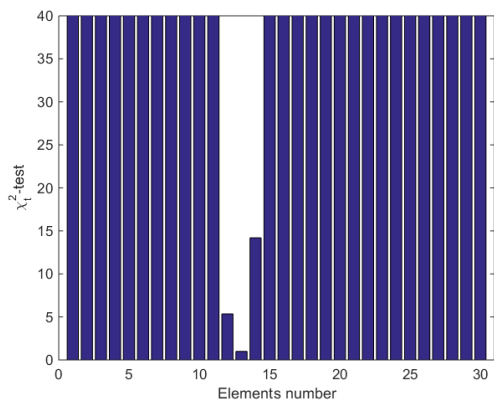

(c) Statistical test

FIGURE 8 With temperature correction - localization results at all elements for stress computation and its statistical evaluation using one dataset and one $s$-value $(s=-1+700 i)$. Damaged elements are 12, 13 and 14 with stiffness loss of $30 \%$.

In Figures 7 a)-7 (c), the values of the theoretical stress, the estimated stress and its statistical evaluation are displayed when temperature is simulated as varying between healthy and damage states, and similarly, the test values (theoretical, estimated stress and its statistical evaluation) are illustrated in Figures $8(a)-8(c)$ when temperature correction is applied. It can be seen that it is difficult to localize the damaged elements correctly at positions 12,13 and 14 in Figure 7, while it is easier to discriminate between the damaged and undamaged elements in Figure 8 In addition, it can also be noticed that the contrast ratio between the test values for the damaged and undamaged elements leads to a higher value for the ratio of the statistical evaluation in Figure 8 when rejecting the temperature variations, compared to Figure 7 .

\section{3 | Performance evaluation of damage localization with and without temperature correction}

To investigate the performance of the damage localization method under temperature variation, the success rate (or probability) of damage localization is evaluated for 100 simulated datasets in a Monte Carlo experiment, with or without using the proposed temperature correction approach. For each dataset, damage localization is seen as successful when the lowest stress or lowest $\chi_{t}^{2}$ value among all elements is indeed at the damaged element. The success rate is then numerically obtained as the percentage of datasets for which the smallest stress or $\chi_{t}^{2}$ value is at the damaged element. The success rate depends on the chosen value for Laplace variable $s$ and serves as the performance indicator for the method.

In order to evaluate the influence of the Laplace variable $s$ on the success rate of the damage localization procedure, each dataset in the Monte-Carlo simulations is evaluated for a set of values of $s$ with different real and imaginary parts in order to obtain the success rate in dependence of $s$. Some of the following plots contain the averaged success rate over several values of $s$. The range of the Laplace variable $s$ has been chosen in the vicinity of the identified poles to reduce the effects of modal truncation in the transfer matrix estimates 26 .

First, the performance of the damage localization approach is evaluated depending on the choice of the value for the Laplace variable $s$. The values of $s$ are chosen in the vicinity of the modes (see Table 1) on a global grid with $\operatorname{Re}(s) \in[-4,-1]$ and 


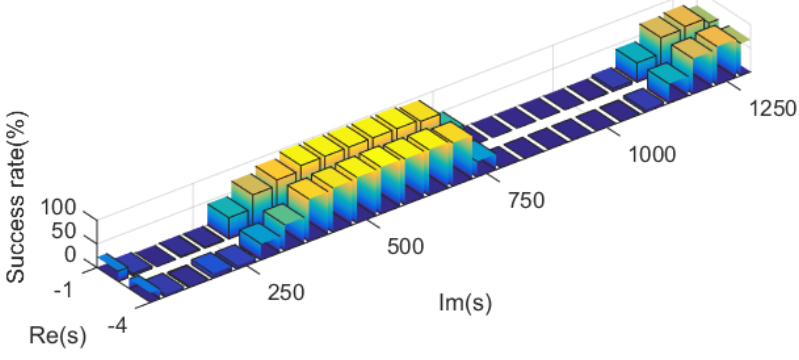

(a) Without temperature correction, using estimated stress.

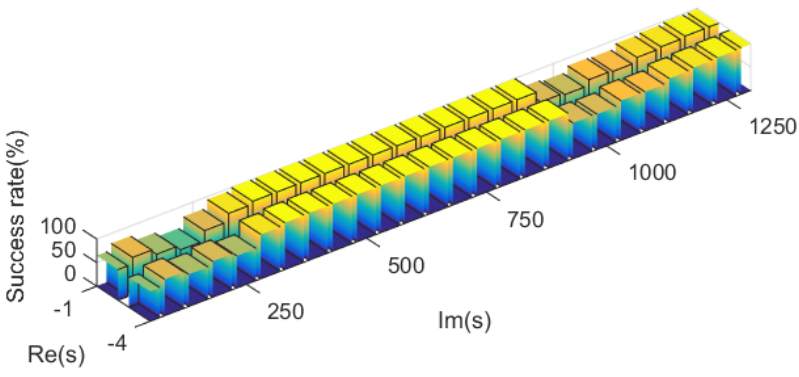

(c) With temperature correction, using estimated stress.

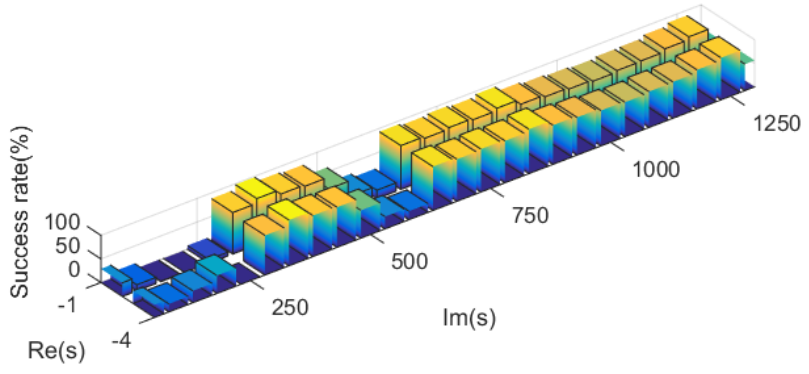

(b) Without temperature correction, using statistical evaluation.

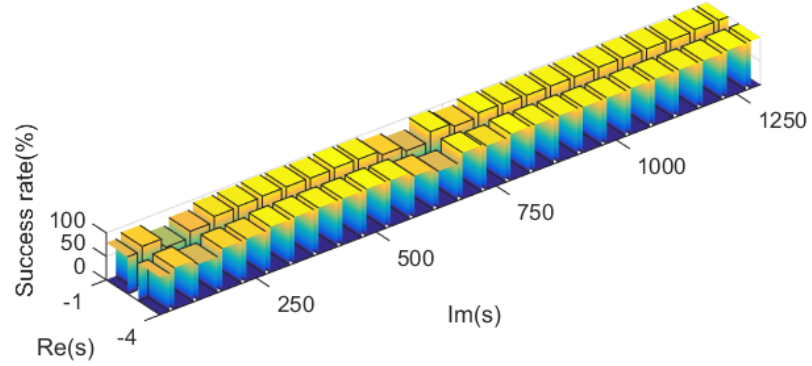

(d) With temperature correction, using statistical evaluation.

FIGURE 9 Damage localization success rates in dependence of $s$. Damaged elements are 12,13 and 14 with stiffness loss of $30 \%$.

$\operatorname{Im}(s) \in[100,1300]$, details are given in 2643 . The success rates of damage localization ( $z$-axis) from the estimated stress and its statistical evaluation are shown in Figure 9 in dependence of the real and imaginary part of the chosen $s$-value ( $x$ and $y$ axis), without and with using the temperature correction approach. In this test case, both the FE model and the healthy datasets are generated at a reference temperature $\left(20^{\circ} \mathrm{C}\right)$ with uniform temperature distribution over the elements of the structure. The damaged dataset is simulated at heat flux $80 \mathrm{~W} \cdot \mathrm{m}^{-2}$. This implies spatial temperature fluctuation over the structure, where the temperature is different for each element of the structure. Both Figures 9(a) 9(b) show that the success rate of the damage localization using either the estimated stress or its statistical evaluation are not satisfying in the entire $s$-plane due to the impact of the temperature variations, while results are significantly improved in Figures 9(c) $9(\mathrm{~d})$ after rejecting the effect of temperature variations. For a better visualization, a comparison of the success rates in the respective range of the Laplace variable $s$ is shown in Figure 10 for a fixed real part $\operatorname{Re}(s)=-1$ (since the choice of the real part of the Laplace variable has little impact on the results). It can be clearly seen that the temperature correction approach significantly improves the damage localization performance nearly everywhere in the $s$-plane in comparison to not using it.

The damage localization success rates with different choices of temperature scenarios in the reference state are shown in Figures 11,13 considering two different types of damages, namely at elements 20 and 21 with $25 \%$ and $50 \%$ stiffness loss, and at elements 12, 13 and 14 with $30 \%$ stiffness loss at each element. In these figures, the mean of the success rates for values of $s$ with $\operatorname{Im}(s)=100,150, \ldots, 1300$ are shown in dependence of the heat flux in the damaged state $(0,20,40,60,80$, and 100 W. $\mathrm{m}^{-2}$ ) with and without the temperature correction approach. Three cases are illustrated:

- Case 1: the FE model and healthy datasets are generated at a reference temperature of $20^{\circ} \mathrm{C}$ with uniform temperature distribution over the elements of the structure,

- Case 2: the FE model and healthy datasets are generated at a reference temperature of $20^{\circ} \mathrm{C}$ with an additional heat flux of $40 \mathrm{~W} . \mathrm{m}^{-2}$, which yields non-uniform temperature distribution over the elements of the structure at reference state,

- Case 3: the FE model and healthy datasets are generated at a reference temperature of $20^{\circ} \mathrm{C}$ with an additional heat flux of $50 \mathrm{~W} \cdot \mathrm{m}^{-2}$. 


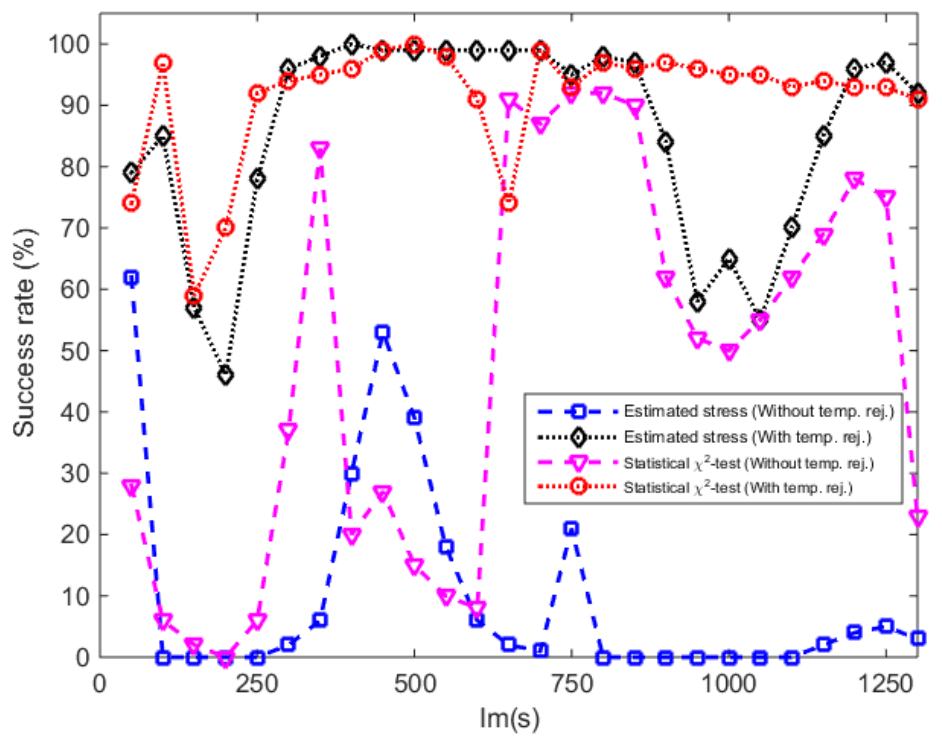

FIGURE $10 \mathrm{With} /$ without temperature correction: Damage localization success rates of the estimated stress and its statistical evaluation in dependence of the value of the Laplace variable $s$. Damaged elements are 12, 13 and 14 with stiffness loss of 30\%.

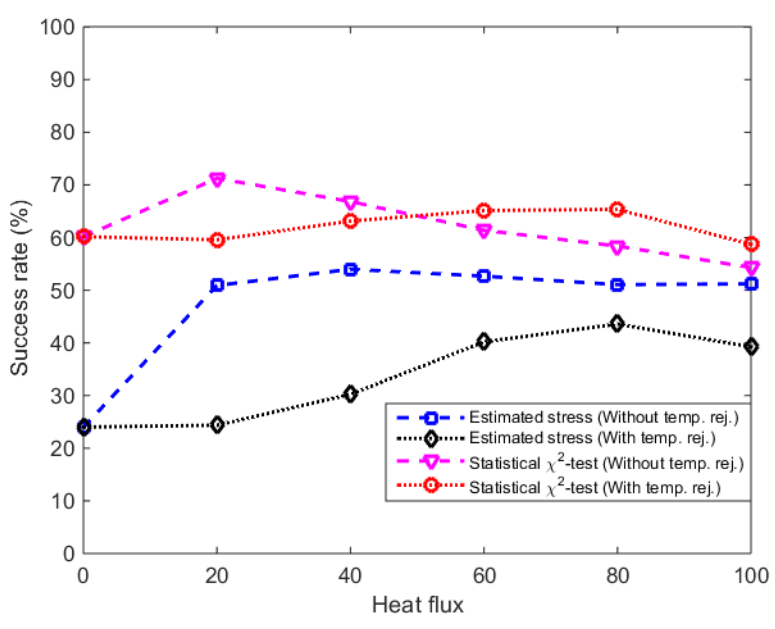

(a) Damage at elements 20 and 21 .

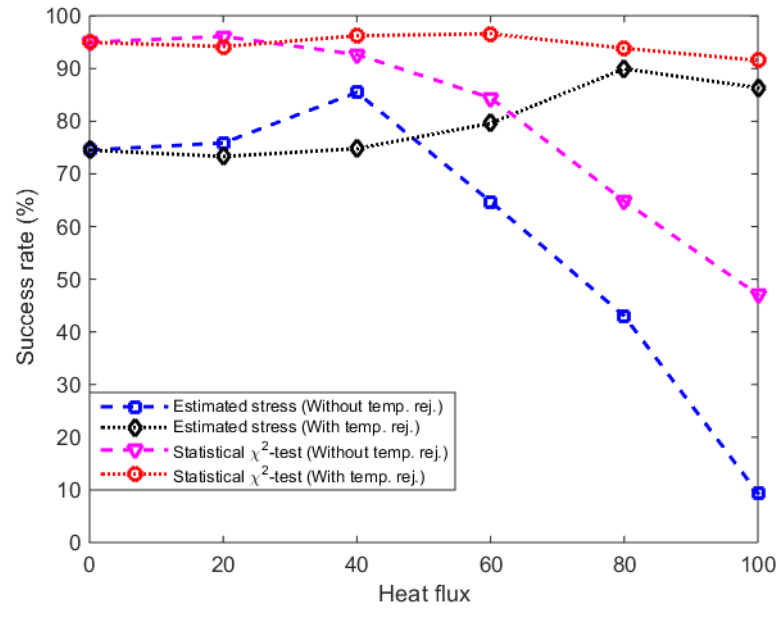

(b) Damage at elements 12,13 and 14 .

FIGURE 11 Damage localization success rates for case 1 (uniform reference temperature at $20^{\circ} \mathrm{C}$ ) with and without temperature correction, in dependence of heat flux in damaged state.

In case 1, Figure 11 shows the success rates of damage localization (based on the estimated stress and its statistical evaluation) for both damage cases. For the first damage case in Figure 11(a) the success rates are in general relatively low and the temperature correction does not influence much the success rates. Without using the temperature correction approach in the second damage case in Figure 11(b), the performance decreases strongly when the heat flux in the damaged state increases. However, with the correction approach, the performance of the localization results is significantly improved in this case, and it can be seen that the success rate is almost constant for the different heat flux in the damaged state. It can also be seen that the performance of the localization - with values of $60 \%$ and $94 \%$, respectively - is now only related to the position of the damage and not dependent on the temperature. 


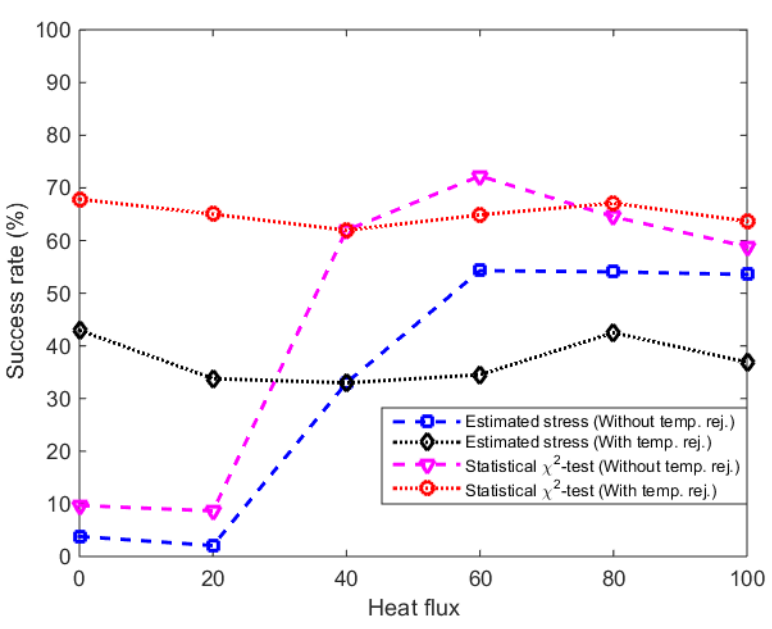

(a) Damage at elements 20 and 21 .

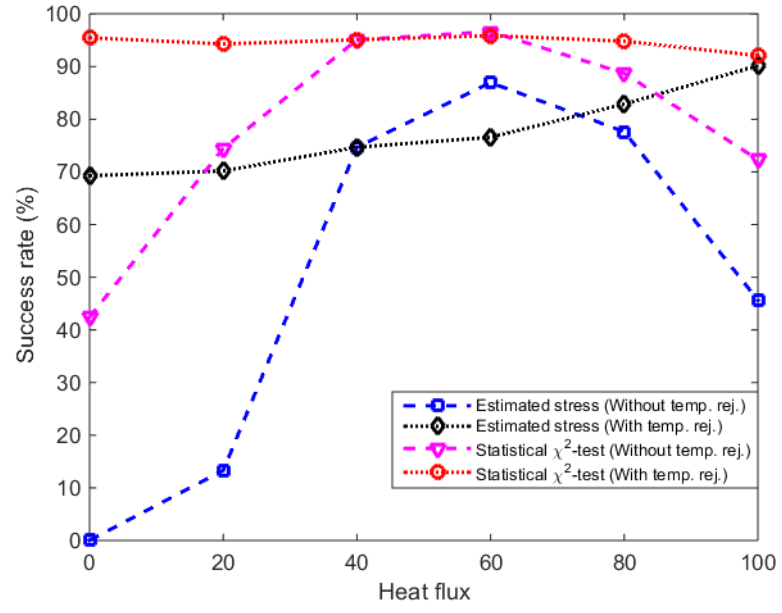

(b) Damage at elements 12,13 and 14

FIGURE 12 Damage localization success rates for case 2 (heat flux $40 \mathrm{~W} \cdot \mathrm{m}^{-2}$ in reference state) with and without temperature correction, in dependence of heat flux in damaged state.

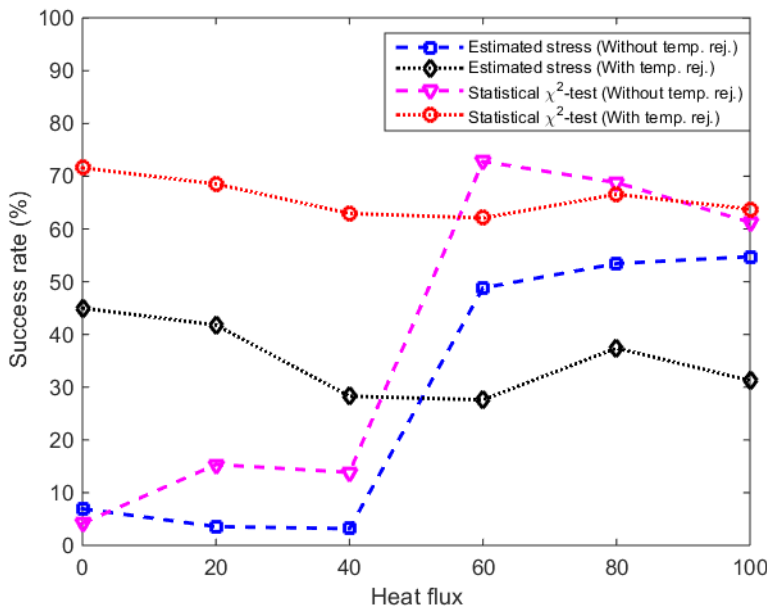

(a) Damage at elements 20 and 21 .

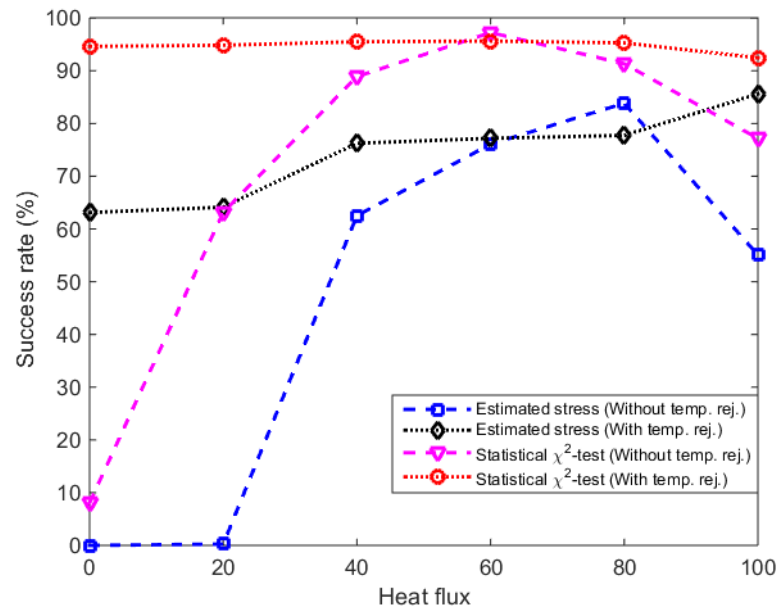

(b) Damage at elements 12, 13 and 14

FIGURE 13 Damage localization success rates for case 3 (heat flux $50 \mathrm{~W} \cdot \mathrm{m}^{-2}$ in reference state) with and without temperature correction, in dependence of heat flux in damaged state.

It is in general expected that the correction of the effect of temperature variation due to heat flux yields a constant damage localization performance, which is no better or worse than performing damage localization in a constant temperature environment. In that sense, the expected outcome of the correction strategy is that the performance should be flat over the different values of the heat flux, not higher.

Cases 2 and 3 correspond to values of the heat flux of 40 and $50 \mathrm{~W} \cdot \mathrm{m}^{-2}$ in the reference state of the structure. In these cases, a non-uniform temperature distribution is generated for both FE model and datasets in the reference state, and data is generated for different values of the heat flux in the damaged state as in case 1. Without the temperature correction, it can be seen that the localization performance is the highest around the reference heat flux, and dropping strongly especially for lower values than the reference heat flux in both damage cases. The performance of the damage localization is significantly improved in both Figures 12 and 13 when the effect of temperature variation has been rejected, where it is quite stable independently of the heat flux in the damaged state. 


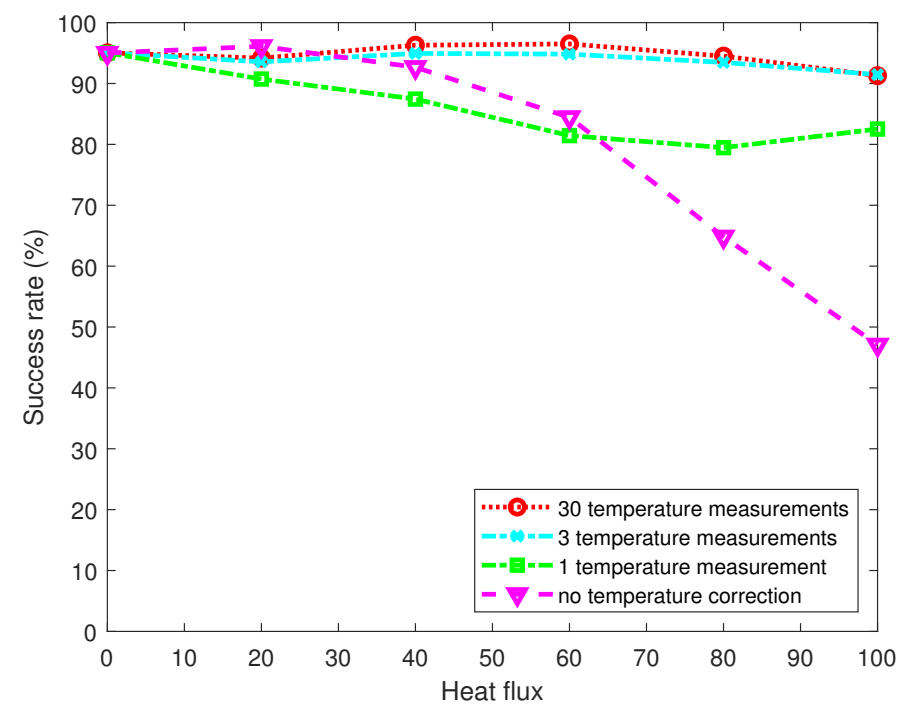

FIGURE 14 Damage localization success rates with statistical evaluation in dependence of the heat flux in the damaged state, using different numbers of temperature measurements for the temperature correction. Damaged elements are 12, 13 and 14 with stiffness loss of $30 \%$.

Finally, the influence of the number of temperature measurements is investigated in the temperature correction approach. So far, the temperatures at all 30 beam elements have been used for the sensitivity-based correction. However, only temperature measurements at fewer locations may be available, e.g. when using temperature sensors instead of an infrared camera in practice. In Figure 14 the performance of the statistical damage localization is presented in the temperature scenario of case 1 (see also Figure 11(b) , but showing also results for less temperature measurements in the temperature correction approach. In particular, only three and one temperature measurements have been used, respectively. In the first case, the temperatures were measured at elements 5,15 and 25, and the temperatures in the elements in between were linearly interpolated, while the temperature in the outer elements was assumed constant. It can be seen that the performance of this case is nearly identical to the case taking all 30 temperatures into account. In the second case, only the temperature at element 15 was considered and assumed constant throughout the beam. In this case, it can be seen that there is a slight drop in performance due to the increased error, however the performance is still better than without temperature correction at high temperature variations.

\section{6 | CONCLUSIONS}

In this paper, a temperature correction strategy has been presented, where the modal parameter estimates are corrected through a sensitivity analysis before applying a subsequent damage localization approach that has been developed previously. The results show that the temperature rejection on the modal parameters is a useful preprocessing step to improve the performance of the damage localization results, making them independent from the current temperature scenario in the damaged state. In all considered cases, it can be concluded that the risk of poor performance of the damage localization method due to the effect of temperature variation can be reduced using this temperature correction strategy, and a significant improvement on the localization success rate is made for non-uniform temperature fields. Since the proposed temperature correction approach operates directly on the modal parameters, it can also be applied to other modal parameter-based damage diagnosis methods.

\section{References}

1. Farrar C, Doebling S, Nix D. Vibration-based structural damage identification. Philosophical Transactions of the Royal Society A: Mathematical, Physical and Engineering Science 2001; 359(1778): 131-149.

2. Carden E, Fanning P. Vibration based condition monitoring: a review. Structural Health Monitoring 2004; 3(4): 355-377. 
3. Balageas D, Fritzen CP, Güemes A. Structural Health Monitoring. London, UK: John Wiley \& Sons . 2010.

4. Fan W, Qiao P. Vibration-based damage identification methods: a review and comparative study. Structural Health Monitoring 2011; 10(1): 83-111.

5. Rytter A. Vibrational based inspection of civil engineering structures. PhD thesis. Aalborg University, Denmark; 1993.

6. Farrar C, Worden K. An introduction to structural health monitoring. Philosophical Transactions of the Royal Society A: Mathematical, Physical and Engineering Sciences 2007; 365(1851): 303-315.

7. Magalhães F, Cunha A, Caetano E. Vibration based structural health monitoring of an arch bridge: From automated OMA to damage detection. Mechanical Systems and Signal Processing 2012; 28: 212-228.

8. Döhler M, Mevel L. Subspace-based fault detection robust to changes in the noise covariances. Automatica 2013; 49(9): 2734-2743.

9. Döhler M, Mevel L, Hille F. Subspace-based damage detection under changes in the ambient excitation statistics. Mechanical Systems and Signal Processing 2014; 45(1): 207-224.

10. Döhler M, Hille F, Mevel L, Rücker W. Structural health monitoring with statistical methods during progressive damage test of S101 Bridge. Engineering Structures 2014; 69: 183-193.

11. Limongelli M, Chatzi E, Döhler M, Lombaert G, Reynders E. Towards extraction of vibration-based damage indicators. 8th European Workshop on Structural Health Monitoring 2016, Bilbao, Spain.

12. Brownjohn JMW, Xia PQ, Hao H, Xia Y. Civil structure condition assessment by FE model updating: methodology and case studies. Finite Elements in Analysis and Design 2001; 37(10): 761-775.

13. Simoen E, De Roeck G, Lombaert G. Dealing with uncertainty in model updating for damage assessment: A review. Mechanical Systems and Signal Processing 2015; 56: 123-149.

14. Friswell M. Damage identification using inverse methods. Philosophical Transactions of the Royal Society A: Mathematical, Physical and Engineering Sciences 2007; 365: 393-410.

15. Gillich G, Ntakpe J, Wahab MA, Praisach Z, Mimis M. Damage detection in multi-span beams based on the analysis of frequency changes. 12th International Conference on Damage Assessment of Structures 2017.

16. Shokrani Y, Dertimanis VK, Chatzi EN, N. Savoia M. On the use of mode shape curvatures for damage localization under varying environmental conditions. Structural Control and Health Monitoring 2018; 25(4): e2132.

17. J. Moughty J, R. Casas J. A state of the art review of modal-based damage detection in bridges: development, challenges, and solutions. Applied Sciences 2017; 7(5): 510.

18. Gu J, Gul M, Wu X. Damage detection under varying temperature using artificial neural networks. Structural Control and Health Monitoring 2017; 24(11): e1998.

19. Eftekhar Azam S, Rageh A, Linzell D. Damage detection in structural systems utilizing artificial neural networks and proper orthogonal decomposition. Structural Control and Health Monitoring 2019; 26(2): e2288.

20. Kim A, Kim H, Lee J. Damage detection of truss structures using two-stage optimization based on micro genetic algorithm. Journal of Mechanical Science and Technology 2014; 28(9): 3687-3695.

21. Shahsavari V, Chouinard L, Bastien J. Wavelet-based analysis of mode shapes for statistical detection and localization of damage in beams using likelihood ratio test. Engineering Structures 2017; 132: 494-507.

22. Lakshmi K, Rao ARM, Gopalakrishnan N. Singular spectrum analysis combined with ARMAX model for structural damage detection. Structural Control and Health Monitoring 2017; 24(9): e1960.

23. Vollmering M, Lenzen A. Theory and numerical application of damage localization method state projection estimation error (SP2E). Structural Control and Health Monitoring 2018; 25(10): e2237. 
24. Balmès E, Basseville M, Mevel L, Nasser H, Zhou W. Statistical model-based damage localization: a combined subspacebased and substructuring approach. Structural Control and Health Monitoring 2008; 15(6): 857-875.

25. Döhler M, Mevel L, Zhang Q. Fault detection, isolation and quantification from Gaussian residuals with application to structural damage diagnosis. Annual Reviews in Control 2016; 42: 244-256.

26. Bernal D. Load Vectors for Damage Location in Systems Identified from Operational Loads. Journal of Engineering Mechanics 2010; 136(1): 31-39.

27. Ulriksen MD, Bernal D, Damkilde L. Shaped input distributions for structural damage localization. Mechanical Systems and Signal Processing 2018; 110: 499-508.

28. Bernal D, Ulriksen MD. Subspace exclusion zones for damage localization. Mechanical Systems and Signal Processing 2019; 114: 120-127.

29. Sohn H. Effects of environmental and operational variability on structural health monitoring. Philosophical Transactions of the Royal Society A: Mathematical, Physical and Engineering Sciences 2007; 365(1851): 539-560.

30. Deraemaeker A, Reynders E, De Roeck G, Kullaa J. Vibration-based structural health monitoring using output-only measurements under changing environment. Mechanical Systems and Signal Processing 2008; 22(1): 34-56.

31. Buren KV, Reilly J, Neal K, Edwards H, Hemez F. Guaranteeing robustness of structural condition monitoring to environmental variability. Journal of Sound and Vibration 2017; 386: 134-148.

32. Liu CY, DeWolf JT. Effect of temperature on modal variability of a curved concrete bridge under ambient loads. Journal of Structural Engineering 2007; 133(12): 1742-1751.

33. Fraser M, Elgamal A, He X, Conte JP. Sensor Network for Structural Health Monitoring of a Highway Bridge. Journal of Computing in Civil Engineering 2010; 24(1): 11-24.

34. Peeters B, Maeck J, Roeck GD. Vibration-based damage detection in civil engineering: excitation sources and temperature effects. Smart Materials and Structures 2001; 10(3): 518-527.

35. Abid SR, Mussa F, Tayşi N, Özakça M. Experimental and finite element investigation of temperature distributions in concrete-encased steel girders. Structural Control and Health Monitoring 2018; 25(1): e2042.

36. Wang D, Liu Y, Liu Y. 3D temperature gradient effect on a steel-concrete composite deck in a suspension bridge with field monitoring data. Structural Control and Health Monitoring 2018; 25(7): e2179.

37. Meruane V, Heylen W. Structural damage assessment under varying temperature conditions. Structural Health Monitoring 2012; 11(3): 345-357.

38. Kullaa J. Structural health monitoring under non-linear environmental or operational influences. Shock and Vibration 2014; 863494.

39. Cross EJ, Worden K, Chen Q. Cointegration: a novel approach for the removal of environmental trends in structural health monitoring data. Proceedings of the Royal Society A: Mathematical, Physical and Engineering Sciences 2011; 467(2133): 2712-2732.

40. Basseville M, Bourquin F, Mevel L, Nasser H, Treyssède F. Handling the temperature effect in vibration monitoring: two subspace-based analytical approaches. Journal of Engineering Mechanics 2010; 136(3): 367-378.

41. Döhler M, Marin L, Bernal D, Mevel L. Statistical decision making for damage localization with stochastic load vectors. Mechanical Systems and Signal Processing 2013; 39(1-2): 426-440.

42. Marin L, Döhler M, Bernal D, Mevel L. Robust statistical damage localization with stochastic load vectors. Structural Control and Health Monitoring 2015; 22: 557-573.

43. Bhuyan M, Döhler M, Lecieux Y, Mevel L, Schoefs F. Statistical damage localization with stochastic load vectors using multiple mode sets. Structural Health Monitoring 2017; 16(5): 518-535. 
44. Peeters B, De Roeck G. Reference-based stochastic subspace identification for output-only modal analysis. Mechanical Systems and Signal Processing 1999; 13(6): 855-878.

45. Döhler M, Mevel L. Fast multi-order computation of system matrices in subspace-based system identification. Control Engineering Practice 2012; 20(9): 882-894.

46. Heylen W, Lammens S, Sas P. Modal Analysis Theory and Testing. Katholieke Universiteit Leuven, Belgium . 1998.

47. Carslaw HS, Jaeger JC. Conduction of heat in solids. London: Oxford University Press. second ed. 1959.

48. Palyvos J. A survey of wind convection coefficient correlations for building envelope energy systems' modeling. Applied Thermal Engineering 2008; 28: 801-808.

49. Hall MR, Dehdezi PK, Dawson AR, Grenfell J, Isola R. Influence of the thermophysical properties of pavement materials on the evolution of temperature depth profiles in different climatic regions. Journal of Materials in Civil Engineering 2012; 24(1): $32-47$.

50. Gartling DK, Reddy JN. The Finite Element Method in Heat Transfer and Fluid Dynamics, Third Edition. CRC series in computational mechanics and applied analysisCRC Press. 3rd ed ed. 2010.

51. Gautier G, Mevel L, Dumoulin J, et al. An experimental setup for subspace based damage identification methods. European Geosciences Union, General Assembly 2018, Vienna, Austria.

52. Crinière A, Dumoulin J, Mevel L. Management of local multi-sensors applied to SHM and long term infrared monitoring: Cloud2IR implementation. Quantitative Infrared Thermography Journal 2019; 16(1): 55-73.

53. EN 1999-1-2. Eurocode 9: Design of aluminum structures - Part 1-2: Structural fire design. 2007. 